\title{
GENE SERPINA1: POTENCIAL MARCADOR DIAGNÓSTICO EM CARCINOMAS PAPILÍFEROS DA TIREOIDE
}

\section{SERPINA1 GENE: POTENTIAL DIAGNOSTIC MARKER IN THYROID PAPILLARY CARCINOMAS}

\author{
Joyce Nascimento Santos ${ }^{1}$ \\ João Paulo da Silva Queiroz ${ }^{2}$ \\ Lázaro Pinto Medeiros Neto ${ }^{3}$ \\ André Bandiera de Oliveira Santos ${ }^{4}$ \\ Renata de Azevedo Canevari ${ }^{5}$
}

\begin{abstract}
Resumo: As neoplasias tireoidianas são o principal tipo de malignidade endócrina, sendo atualmente consideradas um problema de saúde pública com aumento constante em sua incidência nos últimos anos. A descoberta de marcadores moleculares que possam ser aplicados na rotina clínica em conjunto com a biópsia por aspiração com agulha fina (PAAF) pode propiciar um diagnóstico mais preciso e consequentemente um tratamento mais eficiente para o paciente. O objetivo desse estudo foi avaliar se o gene SERPINA1 pode ser considerado um marcador diagnóstico em carcinomas de tireoide. Foi realizada a análise de expressão gênica pela RT-qPCR, em 32 amostras de tecido tireoidiano, sendo 13 bócios, 11 carcinomas papilíferos, quatro carcinomas foliculares e quatro tecidos não tumorais de tireoide. $A$ expressão aumentada do gene SERPINA1 foi observada nas amostras de carcinoma papilífero em comparação com amostras de bócio $(P=0.0319)$ e com as amostras de carcinoma folicular ( $P=0.0430)$. Não foi observada expressão diferencial significativa entre amostras de carcinoma folicular com as amostras de bócio ( $P=0.5329)$. Os resultados da análise de expressão gênica sugerem que o gene SERPINA1 pode ser considerado um marcador diagnóstico em carcinomas papilíferos de tireoide.
\end{abstract}

Palavras-chave: Carcinoma da tireoide. Diagnóstico. Marcador molecular. Expressão gênica. SERPINA1.

Abstract: Thyroid neoplasms are the main type of endocrine malignancy and are currently considered a public health problem with a steady increase in their incidence in recent years. The discovery of molecular markers that can be applied in the clinical routine in conjunction with biopsy by fine needle aspiration (FNAP) can provide a more accurate diagnosis and, consequently, a more efficient treatment for the patient. The aim of this study was to evaluate whether the SERPINA1 gene can be considered a diagnostic marker in thyroid carcinomas. Gene expression analysis by RT-qPCR was performed on 32 samples of thyroid tissue, with 13 goiters, 11 papillary carcinomas, four follicular carcinomas, and four non-tumor thyroid tissues. Increased expression of the SERPINA1 gene was observed in papillary carcinoma samples compared with goiter samples $(P=0.0319)$ and with follicular carcinoma samples $(P=0.0430)$. No

\footnotetext{
1 Universidade do Vale do Paraíba - Univap, E-mail: santosnjoyce@gmail.com.

2 Universidade do Vale do Paraíba - Univap, E-mail: joao.queiroz.ismart@gmail.com.

3 Universidade do Vale do Paraíba - Univap, E-mail: Ipmn 777@yahoo.com.br.

4 Hospital das Clínicas da Faculdade de Medicina da Universidade de São Paulo, E-mail: andre bandiera@yahoo.com.br.

5 Professora Coordenadora do Laboratório de Biologia Molecular do Câncer - IP\&D, Universidade do Vale do Paraíba - UNIVAP, E-mail: rcanevari@univap.br.
} 
significant differential expression was observed between follicular carcinoma samples with goiter samples $(P=0.5329)$. The results of the gene expression analysis suggest that the SERPINA1 gene can be considered a diagnostic marker in papillary thyroid carcinomas.

Keywords: Thyroid carcinoma. Diagnosis. Molecular markers. Gene expression. SERPINA1.

Data de submissão: 11.01 .2021

Data de aprovação: 17.05.2021

Identificação e disponibilidade:

(https://revista.univap.br/index.php/revistaunivap/article/view/2578, http://dx.doi.org/10.18066/revistaunivap.v27i56.2578.

\section{INTRODUÇÃO}

A neuralgia, sintoma mais comum em pacientes acometidos por Herpes zoster $(\mathrm{HZ}), \mathrm{O}$ câncer de tireoide é a mais prevalente neoplasia endócrina, acometendo principalmente mulheres na faixa etária de 20 a 60 anos. No Brasil, essa malignidade é atualmente a oitava mais incidente (GOMES et al., 2011; INCA, 2018, PANEBIANCO et al., 2019), sendo o quinto mais frequente em mulheres (INCA, 2020).

O carcinoma papilífero de tireoide (CPT) é o tipo mais prevalente em comparação às outras lesões (PARK; YOON, 2019), abrangendo de 80 a 90\% de todas as malignidades que acometem a glândula da tireoide (INCA, 2018). A clínica do CPT é lenta e assintomática, apresentando evolução gradual e um bom prognóstico com alta taxa de sobrevida (COELI et al., 2005; LIN; HSUEH; HUANG, 2011). Contudo, o CPT pode apresentar invasão capsular, o que pode favorecer a progressão do tumor e metástase em linfonodos regionais (COELI et al., 2005; LIN; HSUEH; HUANG, 2011; KATOH et al., 2015; PAN et al., 2017; KURE; WADA; NAITO, 2019). Embora o câncer de tireoide tenha um bom prognóstico quando comparado a outras patologias, ainda existem diagnósticos inconclusivos obtidos pelas análises clinicopatológicas aplicadas na rotina.

Dentro os métodos utilizados para a detecção e diagnóstico tumoral das neoplasias da tireoide, destaca-se a ultrassonografia (US) que detecta nódulos até mesmo em estágios subclínicos. Esta técnica é acessível e não invasiva, permitindo a distinção e monitoramento dos nódulos, além de contribuir na detecção das lesões com alto potencial de malignidade. Entretanto, a US não é efetiva na diferenciação dos diferentes tipos de carcinomas (BLANSFIELD; SACK; KUKORA, 2002; RODRIGUES; PONTES; ADAN, 2012; MAROTTA et al., 2016). Atualmente, juntamente com a US é utilizada na rotina clínica a punção por agulha fina (PAAF), sendo considerada a técnica padrão ouro no diagnóstico dessas lesões. Essa metodologia se baseia na coleta, por meio de uma agulha fina, de células e fluidos a serem analisados citopatologicamente (UGURLUOGLU et al., 2015).

No entanto, resultados indeterminados e inconclusivos pela PAAF ocorrem ainda em frequência considerável, podendo atingir cerca de $20 \%$ dos casos investigados (EGSET et al., 2017), podendo inclusive direcionar para tratamento cirúrgico com tireoidectomia total. Deste modo, métodos diagnósticos mais assertivos são necessários para tornar o diagnóstico destes tumores mais precisos e assim contribuir para o prognóstico e terapia mais efetivos para o paciente. 
A identificação de marcadores moleculares diagnósticos pela análise de expressão gênica tem possibilitado a discriminação de nódulos benignos e tecidos tumorais (SADOWSKI; TRIPONEZ, 2019), podendo complementar a análise citológica e histopatológica (FALLAHI et al., 2014). Esta abordagem tem o potencial de auxiliar no diagnóstico precoce, contribuir para o entendimento da progressão tumoral e levar a um tratamento mais direcionado para o paciente (WEBER et al., 2005; PISHKARI et al., 2018). A análise do perfil molecular dos tumores de tireoide como método diagnóstico tem sido implementada na rotina clínica. Em CPT, por exemplo, os marcadores moleculares diagnósticos mais utilizados em rotina são a detecção de mutações nos genes BRAF e RAS, de alterações na expressão das proteínas Erk e p53 e a presença de rearranjos RET/PTC (XING, 2013; OIKONOMOU et al., 2015; VUONG et al., 2017).

Alguns estudos na literatura evidenciaram, por meio da análise de expressão gênica, o potencial diagnóstico do gene SERPINA1 em CPT (JARZAB et al., 2005; ESZLINGER et al., 2007; ZHANG et al., 2019). SERPINA1, também conhecido como alpha-1 antiproteinase, codifica a proteína alfa-1-antitripsina, inibidora de protease serina (VIERLINGER et al., 2011; ZHANG et al., 2019), que estão envolvidas em várias vias, dentre elas, a cascata da coagulação, angiogênese, apoptose e inflamação (LAW et al., 2006). Embora esta proteína tenha sido relacionada a diversas patologias (ABDULLAH et al., 2016; VAJARIA, PATEL, 2017), o mecanismo molecular de sua atuação ainda não está claro.

Considerado a função do gene SERPINA1 em diversas vias relacionadas a carcinogênese e os estudos prévios que sugerem a sua ação no desenvolvimento dos carcinomas de tireoide, o objetivo deste estudo consistiu em avaliar se o gene SERPINA1 possui potencial para ser utilizado como um marcador molecular no diagnóstico das lesões de tireoide.

\section{MATERIAL E MÉTODOS}

Esse estudo foi aprovado pelo Comitê de Ética em Pesquisa da Universidade do Vale do Paraíba (nº 221.402/2013), que segue a resolução CNS/MS 196/96. Trinta e duas amostras de tecido tireoidiano, sendo 13 amostras de bócio, 11 amostras de CPT, quatro amostras de CFT (carcinoma folicular de tireoide) e quatro amostras de tecido não tumoral de tireoide, constituindo o grupo normalizador da RTq-PCR, foram obtidas por tireoidectomia total com cada amostra correspondendo a um paciente cirurgiado, na Universidade de São Paulo, Instituto do Câncer do Estado de São Paulo (ICESP), juntamente com o Hospital das Clínicas da Faculdade de Medicina de São Paulo, Brasil.

Os tecidos utilizados foram analisados histopatologicamente, seguindo os critérios de diagnóstico da Sociedade Brasileira de Patologia para câncer de tireoide e foram utilizadas apenas as amostras com a presença de no mínimo $80 \%$ de células tumorais no fragmento de tecido tireoidiano obtido.

\subsection{EXTRAÇÃO DE RNA E SÍNTESE DE cDNA}

Esse estudo foi aprovado pelo Comitê de Ética em Pesquisa da Universidade do Vale do Paraíba (nº 221.402/2013), que segue a resolução CNS/MS 196/96. Trinta e duas amostras de tecido tireoidiano, sendo 13 amostras de bócio, 11 amostras de CPT, quatro amostras de CFT (carcinoma folicular de tireoide) e quatro amostras de tecido não tumoral de tireoide, constituindo o grupo normalizador da RTq-PCR, foram 
obtidas por tireoidectomia total com cada amostra correspondendo a um paciente cirurgiado, na Universidade de São Paulo, Instituto do Câncer do Estado de São Paulo (ICESP), juntamente com o Hospital das Clínicas da Faculdade de Medicina de São Paulo, Brasil.

Os tecidos utilizados foram analisados histopatologicamente, seguindo os critérios de diagnóstico da Sociedade Brasileira de Patologia para câncer de tireoide e foram utilizadas apenas as amostras com a presença de no mínimo $80 \%$ de células tumorais no fragmento de tecido tireoidiano obtido.

\subsection{ANÁLISE QUANTITATIVA EM TEMPO REAL}

A detecção de análise de expressão gênica pela análise quantitativa em tempo real (RT-qPCR) foi realizada no equipamento ABI PRISM 7500 Sequence Detection System (Life Tchnologies, USA) usando o reagente Platinum SYBR Green Rt-qPCR Super Mix UDG (Applied Biosystems, Life Tchnologies, Carlsbad, CA). Os iniciadores para a amplificação do gene alvo SERPINA1 e do gene endógeno PPIA (peptidil prolil isomerase A) determinados usando o software Primer Express (versão3.0, PE Applied Biosystems, Foster City, CA, USA) foram: 5' -AAGGAGCTTGACAGAGACACAGTTT3' (forward) e 5'-GGTCTCTCCCATTTGCCTTTAA-3' (reverse) para o SERPINA1 e 5'GCACTGGAGAGAAAGGATTTGG-3' (forward) e TCACCACCCTGACACATAAACC-3' (reverse) para o gene PPIA. Para evitar a amplificação de uma possível contaminação com DNA genômico, os iniciadores foram desenhados na junção entre dois exons ou em exons diferentes.

Para ambos os genes SERPINA1 e PPIA foram realizadas as curvas padrões e obtidos os valores de slope, que possibilitam o cálculo de eficiência de amplificação dos iniciadores e a definição de diluição a ser utilizada $(100,20,4,0.8$, e $0.16 \mathrm{ng} / \mu \mathrm{l})$. Ambos os conjuntos de iniciadores mostraram eficiência de amplificação superior a 90\%. (Sequence Detection System software, versão 2.1, PE Applied Biosystems). A curva de dissociação também foi realizada para ambos os genes e mostraram especificidade dos iniciadores utilizados e ausência de formação de primers dimers. Os thresholds foram definidos em todas as análises de RT-qPCR.

A análise de expressão relativa do gene alvo SERPINA1 foi realizada pelo método Delta-Delta $\mathrm{Ct}(\Delta \Delta \mathrm{Ct})$, onde o valor médio de Cts (cyclethreshold) obtido para o gene alvo foi comparado com a média do Cts do gene endógeno e, posteriormente, normalizado pela média das amostras normais. Os valores de QR (quantificação relativa) que consiste no valor obtido da expressão do gene normalizado no grupo experimental divido pela expressão do gene normalizado no grupo controle, foi calculado para cada gene analisado pela fórmula $2^{\wedge}$ (- Delta Delta $\mathrm{Ct}$ ). Os valores de QR menores ou iguais a $0.5(\mathrm{QR} \leq 0.5)$ indicam diminuição de expressão do gene alvo e os valores de $Q R$ superiores a $2.0(Q R>2.0)$ indicam aumento de expressão do gene alvo.

A análise estatística dos dados da RT-qPCR foi realizada utilizando o programa GraphPad Prism In Stat (versão 6.00, San Diego, CA, EUA), incluindo o teste não paramétrico de Mann-Whitney para a determinação significativa entre os grupos analisados. Os resultados foram considerados significativamente estatísticos com valores de $p \leq 0.05$.

\section{RESULTADOS}

Os valores de expressão do gene SERPINA1 nas 32 amostras de lesões de 
tireoide comparadas com o grupo de amostras não tumorais (grupo normalizador) estão descritos na Tabela 1. No grupo de amostras de CPT, duas amostras apresentaram diminuição de expressão $(Q R=0,00$ e 0,09$)$ e nove amostras apresentaram aumento de expressão $(Q R=3,83$ a 4004,76$)$ em relação às amostras não tumorais. Nas amostras de CFT, quatro amostras apresentaram diminuição de expressão ( $Q R=0,03$ a 0,35) e nenhuma amostra apresentou aumento de expressão em comparação às amostras de tecido não tumoral (Tabela 1).

Tabela 1 - Valores de expressão relativa do gene SERPINA1 nas amostras de lesões de tireoide comparadas com o grupo de amostras não tumorais (grupo normalizador).

\begin{tabular}{ccc}
\hline AMOSTRA & DIAGNÓSTICO & VALORES DE QR \\
\hline 1 & Bócio & $\mathbf{1 0 7 3 , 0 5}$ \\
2 & Bócio & $\mathbf{8 7 , 8 8}$ \\
3 & Bócio & $\mathbf{2 , 4 9}$ \\
4 & Bócio & $\mathbf{0 , 6 3}$ \\
5 & Bócio & $\mathbf{0 , 0 9}$ \\
6 & Bócio & $\mathbf{0 , 0 2}$ \\
7 & Bócio & $\mathbf{0 , 0 9}$ \\
8 & Bócio & $\mathbf{0 , 1 4}$ \\
9 & Bócio & $\mathbf{0 , 1 3}$ \\
10 & Bócio & $\mathbf{0 , 2 5}$ \\
11 & Bócio & $\mathbf{0 , 1 7}$ \\
12 & Bócio & $\mathbf{0 , 0 2}$ \\
13 & Bócio & $\mathbf{0 , 4 5}$ \\
14 & CPT & $\mathbf{2 9 , 2 9}$ \\
15 & CPT & $\mathbf{8 3 0 , 3 1}$ \\
16 & CPT & $\mathbf{3 , 8 3}$ \\
17 & CPT & $\mathbf{5 0 0 , 5 9}$ \\
18 & CPT & $\mathbf{5 6 1 , 2 5}$ \\
19 & CPT & $\mathbf{1 7 , 4 2}$ \\
20 & CPT & $\mathbf{4 0 0 4 , 7 6}$ \\
21 & CPT & $\mathbf{2 4 7 3 , 7 8}$ \\
22 & CPT & $\mathbf{4 , 8 3}$ \\
23 & CPT & $\mathbf{0 , 0 0}$ \\
24 & CPT & $\mathbf{0 , 0 9}$ \\
25 & CFT & $\mathbf{0 , 0 6}$ \\
26 & CFT & $\mathbf{0 , 0 3}$ \\
27 & CFT & $\mathbf{0 , 2 7}$ \\
28 & CFT & $\mathbf{0 , 3 5}$ \\
\hline
\end{tabular}

Legenda: $Q R$ : quantificação relativa. $Q R \leq 0,5$ : diminuição de expressão gênica em relação ao tecido não tumoral. QR > 2,0: aumento de expressão gênica em relação ao tecido não tumoral. CPT: carcinoma papilífero de tireoide. CFT: carcinoma folicular de tireoide.

Fonte: Elaborado pelos autores (2020).

Três comparações foram realizadas utilizando os valores de QR obtidos pela análise de expressão gênica: CPT versus bócio (Análise 1), CFT versus bócio (Análise 2) e CPT versus CFT (Análise 3). $\mathrm{Na}$ análise 1 foi observada diferença estatisticamente significativa, com aumento de expressão nas amostras de CPT em relação às amostras de bócio $(P=0.0319)$ (Teste de Mann-Whitney). Na análise 2 não foi observada significância estatística para o gene SERPINA1 $(P=0.5329)$. Na análise 3 foi observado diferença estatisticamente significativa, com aumento de expressão 
nas amostras de CPT em relação às amostras de CFT $(P=0.0430)$ (Figura 1$)$.

Figura 1 - Comparação entre as medianas dos níveis de expressão do gene SERPINA1. (A) Análise 1: CPT versus bócio, (B) Análise 2: CFT versus bócio e (C): Análise 3: CFT versus CPT.
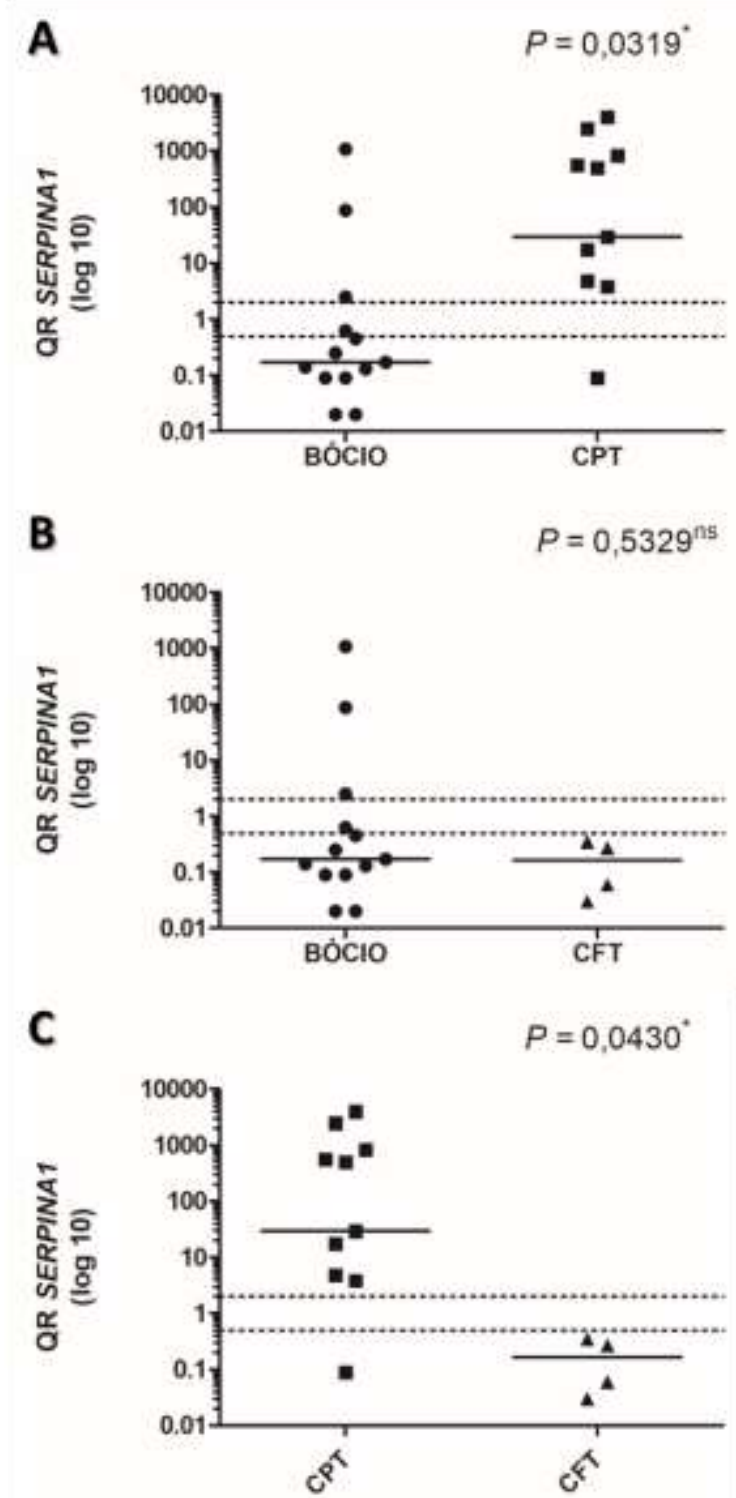

Legenda: QR: quantificação relativa do gene. CPT: carcinoma papilífero de tireoide. CFT: carcinoma folicular de tireoide.

Fonte: Elaborado pelos Autores (2020).

\section{DISCUSSÃO}

No presente estudo, os níveis de expressão do gene SERPINA1 em amostras de CPT foram significativos e concordantes com os resultados encontrados nas análises de expressão deste gene realizados por estudos prévios da literatura. Estes estudos o descobriram como um importante marcador diagnóstico em CPT e o relacionaram com os processos de adesão celular, matriz extracelular, angiogênese e metástase (HUANG et al., 2001; JARZAB et al., 2005; ESZLINGER et al., 2007; VIERLINGER et al., 2011, ZHANG et al., 2019). Além disso, este gene também foi descrito como marcador diagnóstico em outros tumores, tais como: carcinoma 
mamário (LAURSEN; LYKKESFELDT, 1992), hepatocelular (ORDÓÑEZ; MANNING JR, 1984), gástrico (NISHINO et al., 1988) e câncer de pulmão (HIGASHIYAMA et al., 1995).

Estudos prévios já indicam o potencial diagnóstico do gene SERPINA1 em amostras de CPT. Huang et al. (2001) observaram, pela técnica microarray, aumento de expressão deste gene em oito amostras de CPT na população da América do Norte, sendo o resultado validado pela análise de RT-qPCR e por imuno-histoquímica em amostras adicionais, sugerindo assim o potencial diagnóstico deste gene em CPT. Jarzab et al. (2005), por meio da análise de microarray e validação pela RT-qPCR, mostraram que o gene SERPINA1 é um potente marcador para CPT na população polonesa. Qu et al. (2016) detectaram, pela análise de microarray, 167 genes diferencialmente expressos em amostras de CPT, sendo o gene SERPINA1 apresentando expressão aumentada em comparação com os tecidos normais da glândula da tireoide. Teng et al. (2018), com o objetivo de facilitar o prognóstico e estratégias terapêuticas, identificaram por meio de 242 perfis de expressão de CPT, uma assinatura transcriptômica associada à agressividade do CPT, onde o gene SERPINA1 aparece dentre 10 potenciais biomarcadores. A expressão elevada deste gene nos tecidos de CPT também foi observada por Vierlinger et al. (2011), que utilizaram uma meta-análise que identificou o SERPINA1 como capaz de discriminar com alta precisão os tecidos de CPT quando comparados com as lesões benignas ou com os tecidos não tumorais, possuindo $99 \%$ de acurácia na diferenciação desses tumores em relação às lesões benignas, como por exemplo, o bócio adenomatoso ou coloide. Zhang et al. (2019) pela análise do gene SERPINA1 pela RT-qPCR em 24 tecidos de tireoide na população chinesa também correlacionaram este gene com o desenvolvimento de nódulos tireoidianos. Em nosso estudo, também podemos observar o alto potencial do gene SERPINA1 na diferenciação das amostras de CPT de bócio ( $P=0.0319)$ e de CFT $(P=0.0430)$.

Em alguns estudos, a avaliação proteica do produto do gene SERPINA1 também tem sido realizada e o aumento da expressão desta proteína em CPT tem sido observada (POBLETE et al. 1996; LAl et al. 1998), assim como o aumento da expressão gênica já evidenciado. Poblete et al. (1996), utilizando a técnica de imunohistoquímica e Western Blot, concluíram que a proteína e o gene SERPINA1 são potenciais marcadores no diagnóstico em CPT. Lai et al. (1998) relataram em CPT, a elevada expressão da proteína alfa-1-antitripsina, codificada pelo gene SERPINA1, e a ausência da expressão desta proteína nos tecidos tireoidianos não tumorais, sugerindo esta proteína como um marcador molecular para essa variação histológica.

\section{CONCLUSÃO}

Os resultados da análise de expressão gênica demonstraram que o gene SERPINA1 apresentou alto poder de diferenciação entre CPT e bócio, e entre os tumores malignos CPT e CFT, sugerindo o potencial deste gene como marcador diagnóstico de CPT na população brasileira. A identificação desse gene como marcador diagnóstico dessas lesões pode auxiliar no diagnóstico precoce, na triagem de lesões não manifestadas clinicamente, além de colaborar com a seleção da melhor conduta cirúrgica e possivelmente no tratamento adjuvante de lesões confirmadas como malignas na população brasileira. Estudos adicionais com um maior número de amostras são necessários para validar esses resultados e a pesquisa de outros marcadores moleculares são importantes para discriminar efetivamente o diagnóstico dessas lesões. 


\section{REFERÊNCIAS}

ABDULLAH, $\mathrm{M}$ et al. Tissue and serum samples of patients with papillary thyroid cancer with and without benign background demonstrate different altered expression of proteins. Peer J., v. 4, p. e2450, 2016.

BLANSFIELD, J. A.; SACK, M. J.; KUKORA, J. S. Recent experience with preoperative fine-needle aspiration biopsy of thyroid nodules in a community hospital. Arch. surg., v. 137, n. 7, p. 818-821, 2002.

COELI, C. M. et al. Incidência e mortalidade por câncer de tireóide no Brasil. Arquivos Brasileiros de Endocrinologia \& Metabologia, v. 49, n. 4, p. 503-509, 2005. EGSET, A. V. et al. Risk of malignancy in fine-needle aspiration biopsy in patients with thyroid nodules. Dan. Med. J., v. 64, n. 2, 2017.

ESZLINGER, M. et al. Perspectives and limitations of microarray-based gene expression profiling of thyroid tumors. Endocr. rev., v. 28, n. 3, p. 322-338, 2007.

FALLAHI, P. et al. Molecular Diagnostics of Fine Needle Aspiration for the Presurgical Screening of Thyroid Nodules. Current Genomics, v. 15, n. 3, p. 171-177, 2014.

GOMES, E. M. S. et al. Frequency of thyroid carcinoma and thyroid autoimmunity in first- degree relatives of patients with papillary thyroid carcinoma - A single center experience. Arq. Bras. Endocrinol. Metab., v. 55, n. 5, p. 326-330, 2011.

HIGASHIYAMA, M. et al. Alpha-1-antichymotrypsin expression in lung adenocarcinoma and its possible association with tumor progression. Cancer, v. 76, n. 8, p. 1368-1376, 1995.

HUANG, Y. et al. Gene expression in papillary thyroid carcinoma reveals highly consistent profilesProc. Natl. Acad. Sci. U. S. A., v. 98, n. 26, p. 15044-15049, 2001.

INCA. Estimativa 2018. Incidencia de cáncer no Brasil. [s.l: s.n.], 2018

INCA. Estimativa 2020. Incidência de Câncer no Brasil. [s.l: s.n.], 2020.

JARZABB, B. et al. Gene expression profile of papillary thyroid cancer: Sources of variability and diagnostic implications. Cancer res., v. 65, n. 4, p. 1587-1597, 2005.

$\mathrm{KATOH}, \mathrm{H}$. et al. Classification and general considerations of thyroid Cancer. Ann.

Clin. Pathol., v. 3, n. 1, p.1-9, 2015.

KURE, S.; WADA, R.; NAITO, Z. Relationship between genetic alterations and clinicopathological characteristics of papillary thyroid carcinoma. Med. Mol. Morphol., v. 52, n. 4, p. 181-186, 2019.

LAI, M. L. et al. Alpha-1-antichymotrypsin immunoreactivity in papillary carcinoma of the thyroid gland. Histopathology, v. 33, n. 4, p. 332-336, 1998. 
LAURSEN, I.; LYKKESFELDT, A. E. Purification and characterization of an a1antichymotrypsin-like $66 \mathrm{kDa}$ protein from the human breast cancer cell line, MCF-7. Biochim. biophys. acta, v. 1121, n. 1-2, p. 119-129, 1992.

LAW, R. H. P. et al. An overview of the serpin superfamily. Genome biol., v. 7, n. 5, p. 1-11, 2006.

LIN, J.-D.; HSUEH, C.; HUANG, B.-Y.. Papillary thyroid carcinoma with different histological patterns. Chang gung med. J., v. 34, n. 1, p. 23-34, 2011.

MAROTTA, V. et al. Application of molecular biology of differentiated thyroid cancer for clinical prognostication. Endocr. Relat. cancer., v. 23, n. 11, p. R499-R515, 2016.

NISHINO, N. et al. The urokinase type of plasminogen activator in cancer of digestive tracts. Thromb. res., v. 50, n. 4, p. 527-535, 1988.

OIKONOMOU, E. et al. BRAF vs RAS oncogenes: Are mutations of the same pathway equal? Differential signalling and therapeutic implications. Oncotarget, v. 5, n. 23, 2015.

ORDÓÑEZ, N. G.; MANNING JR, J.T. Comparison of $\alpha-1-A n t i t r y p s i n$ and $\alpha-1$ Antichymotrypsin in Hepatocellular Carcinoma: An Immunoperoxidase Study. Am. J. gastroenterol., v. 79, n. 12, 1984.

PAN, D. H. et al. The diagnostic and prognostic values of Ki-67/MIB-1 expression in thyroid cancer: a meta-analysis with 6,051 cases. Onco Targets Ther., v. 10, p. 3261-3276, 2017.

PANEBIANCO, F. et al. Spectrum of TERT promoter mutations and mechanisms of activation in thyroid cancer. Cancer Med., v. 8, n. 13, p. 5831-5839, 2019.

PARK, J. H.; YOON, J. H. Lobectomy in patients with differentiated thyroid cancer: Indications and follow-up. Endocr. Relat. cancer, v. 26, n. 7, p. R381-R393, 2019.

PISHKARI, S. et al. The role of microRNAs in different types of thyroid carcinoma: a comprehensive analysis to find new miRNA supplementary therapies. J. Endocrinol. Invest., v. 41, n. 3, p. 269-283, 2018.

POBLETE, M.T. et al. Alpha 1-antitrypsin expression in human thyroid papillary carcinoma. Am. J. Surg. Pathol., v. 20, n. 3, p. 956-963, 1996.

QU, T. et al. Identification of potential biomarkers and drugs for papillary thyroid cancer based on gene expression profile analysis. Mol. Med. Rep., v. 14, n. 6, p. 5041-5048, 2016.

RODRIGUES, H. G. C.; PONTES, A. A. N.; ADAN, L. F. F. Use of molecular markers in samples obtained from preoperative aspiration of thyroid. Endocr. J., v. 59, n. 5, p. 417-424, 2012. 
SADOWSKI, S. M.; TRIPONEZ, F. Diagnosis and management of indeterminate thyroid nodules. Gland Surg., v. 8, n. Suppl 2, p. S60-S61, 2019.

TENG, H. et al. Transcriptomic signature associated with carcinogenesis and aggressiveness of papillary thyroid carcinoma. Theranostics, v. 8, n. 16, p. 43454358, 2018.

UGURLUOGLU, C. et al. Fine needle aspiration biopsy of thyroid nodules: cytologic and histopathologic correlation of 1096 patients. Int. J. Clin. Exp. Pathol., v. 8, n. 11, p. $14800,2015$.

VAJARIA, B.N.; PATEL, P.S. Glycosylation: a hallmark of cancer? Glycoconj. J., v. 34, n. 2, p. 147-156, 2017.

VIERLINGER, K. et al. Identification of SERPINA1 as single marker for papillary thyroid carcinoma through microarray meta analysis and quantification of its discriminatory power in independent validation. BMC Med. Genomics, v. 4, n. 1, p. 30, 2011.

VUONG, H. G. et al. Prognostic implication of BRAF and TERT promoter mutation combination in papillary thyroid carcinoma-A meta-analysis. Clin. endocrinol., v. 7, n. 5, p. 411-417, 2017.

WEBER, F. et al. Genetic classification of benign and malignant thyroid follicular neoplasia based on a three-gene combination. J. clin. endocrinol. metab., v. 90, n. 5, p. 2512-2521, 2005.

XING, M. Molecular pathogenesis and mechanisms of thyroid cancer. Nat. rev., v. 13, p. 184-199, 2013.

ZHANG, K. et al. Identification and validation of potential target genes in papillary thyroid cancer. Eur. j. pharmacol., v. 843, n. November 2018, p. 217-225, 2019. 